\title{
QUASI GENERALIZED OPEN SETS AND QUASI GENERALIZED CONTINUITY ON BIGENERALIZED TOPOLOGICAL SPACES
}

\author{
Won Keun Min and Young Key Kim
}

\begin{abstract}
We introduce the notions of bigeneralized topological spaces and quasi generalized open sets, and study some basic properties for the sets. We also introduce the notion of quasi generalized continuity on bigeneralized topological spaces, and investigate characterizations for the continuity.
\end{abstract}

\section{Introduction}

In [1], Császár introduced the notions of generalized neighborhood systems and generalized topological spaces. He also introduced the notions of continuous functions and associated interior and closure operators on generalized topological spaces in [1]. The concept of bitopological spaces was introduced by Kelly [3]. A set equipped with two topologies is called a bitopological space. Datta [2] has states that a subset $S$ of bitopological space $(X, P, Q)$ is quasiopen if for every $x \in S$, there exists a $P$-open set $U$ such that $x \in U \subseteq S$, or a $Q$-open set $V$ such that $x \in V \subseteq S$.

The purpose of this paper is to introduce and investigate the notion of quasi generalized open sets defined by using two generalized topologies instead of two topologies. We also introduce the notion of quasi generalized continuity between bigeneralized topological spaces, and investigate characterizations for the continuity.

\footnotetext{
${ }^{0}$ Corresponding Author : wkmin@kangwon.ac.kr

Received September 6, 2010. Accepted November 8, 2010.

2000 Mathematics Subject Classification: 54A05, 54C05.

Key words and phrases: generalized topological space, bigeneralized topological space, quasi generalized open sets, quasi generalized continuous function.
} 


\section{Preliminaries}

We recall some notions and notations defined in [1]. Let $X$ be a nonempty set and $\psi$ be a collection of subsets of $X$. Then $\psi$ is called a generalized topology (briefly GT) on $X$ iff $\emptyset \in \psi$ and $G_{i} \in \psi$ for $i \in I \neq \emptyset$ implies $G=\cup_{i \in I} G_{i} \in \psi$. We call the pair $(X, \psi)$ a generalized topological space (briefly GTS) on $X$. The elements of $\psi$ are called $\psi$-open sets and the complements are called $\psi$-closed sets.

If $\psi$ is a generalized topology on $X$ and $A \subseteq X$, the interior of $A$ (denoted by $i_{\psi}(A)$ ) is the union of all $G \subseteq A, G \in \psi$, and the closure of $A$ (denoted by $\left.c_{\psi}(A)\right)$ is the intersection of all $\psi$-closed sets containing A.

Let $\psi$ and $\psi^{\prime}$ be generalized topologies on $X$ and $Y$, respectively. Then a function $f:(X, \psi) \rightarrow\left(Y, \psi^{\prime}\right)$ is said to be $\left(\psi, \psi^{\prime}\right)$-continuous [1] if $G^{\prime} \in \psi^{\prime}$ implies that $f^{-1}\left(G^{\prime}\right) \in \psi$.

Theorem 2.1. ([1]) Let $(X, \psi)$ be generalized topological space. Then

(1) $c_{\psi}(A)=X-i_{\psi}(X-A)$

(2) $i_{\psi}(A)=X-c_{\psi}(X-A)$.

\section{Quasi generalized open sets in bigeneralized topological spaces}

Definition 3.1. Let $X$ be a nonemptyset and $\psi_{1}, \psi_{2}$ generalized topologies on $X$. A triple $\left(X, \psi_{1}, \psi_{2}\right)$ is called a bigeneralized topological space (briefly biGTS).

Throughout the present paper, $\left(X, \psi_{1}, \psi_{2}\right),\left(Y, \mu_{1}, \mu_{2}\right)$ and $\left(Z, \lambda_{1}, \lambda_{2}\right)$ denote bigeneralized topological spaces.

Remark 3.2. In a bigeneralized topological space $\left(X, \psi_{1}, \psi_{2}\right)$, if the generalized topologies $\psi_{1}, \psi_{2}$ are topologies on $X$, then the bigeneralized topological space $\left(X, \psi_{1}, \psi_{2}\right)$ is a bitopological space introduced by Kelly in [3].

Definition 3.3. Let $\left(X, \psi_{1}, \psi_{2}\right)$ be a biGTS. A subset $A$ of $X$ is said to be quasi $\left(\psi_{1}, \psi_{2}\right)$-open (briefly quasi $q_{\psi}$-open) if for every $x \in A$, there exists a $\psi_{1}$-open set $U$ such that $x \in U \subseteq A$, or a $\psi_{2}$-open set $V$ such that $x \in V \subseteq A$. A subset $A$ of $X$ is said to be quasi $q_{\psi}$-closed if the complement of $A$ is quasi $q_{\psi}$-open. 
In a given bigeneralized topological space $\left(X, \psi_{1}, \psi_{2}\right)$, a quasi $q_{\psi}$-open set need not be $\psi_{1}$-open or $\psi_{2}$-open as shown in the next example.

Example 3.4. Let $X=\{a, b, c, d\}$. Consider two generalized topologies $\psi_{1}=\{\emptyset,\{a, b\}\}$ and $\psi_{2}=\{\emptyset,\{a, c\}\}$ on $X$. Then $\{a, b, c\}$ is a quasi $q_{\psi}$-open set but it is neither $\psi_{1}$-open nor $\psi_{2}$-open

Lemma 3.5. Let $\left(X, \psi_{1}, \psi_{2}\right)$ be a biGTS and $A$ a subset of $X$. Then

(1) $A$ is quasi $q_{\psi^{-}}$open if and only if $A$ is a union of a $\psi_{1}$-open set and a $\psi_{1}$-open set.

(2) $A$ is quasi $q_{\psi}$-closed if and only if $A$ is a intersection of a $\psi_{1}$-closed set and a $\psi_{1}$-closed set.

(3) Any union of quasi $q_{\psi^{-}}$open sets is quasi $q_{\psi^{-} \text {-open }}$

Proof. Obvious.

Remark 3.6. In a given bigeneralized topological space, the intersection of two quasi $q_{\psi}$-open sets may not be quasi $g$-open as shown in the next example.

Example 3.7. Let $X=\{a, b, c, d\}$. Consider two generalized topologies $\psi_{1}=\{\emptyset,\{d\},\{a, b\},\{a, b, d\}\}$ and $g_{2}=\{\emptyset,\{c\}\{a, d\},\{a, c, d\}\}$ on $X$. Then $\{a, b, c\}$ and $\{a, c, d\}$ are quasi $q_{\psi}$-open sets but the intersection $\{a, c\}$ is not quasi $q_{\psi}$-open set.

Definition 3.8. Let $\left(X, \psi_{1}, \psi_{2}\right)$ be a biGTS and $A \subseteq X$. We define the quasi $q_{\psi}$-closure (briefly $c_{q_{\psi}}(A)$ ) and the quasi $q_{\psi}$-interior (briefly $\left.i_{q_{\psi}}(A)\right)$ as the following:

$$
\begin{gathered}
c_{q_{\psi}}(A)=\cap\left\{F: A \subseteq F \text { for a quasi } q_{\psi} \text {-closed set } F\right\} ; \\
i_{q_{\psi}}(A)=\cup\left\{G: G \subseteq A \text { for a quasi } q_{\psi} \text {-open set } G\right\} .
\end{gathered}
$$

Let $\left(X, \psi_{1}, \psi_{2}\right)$ be a biGTS and $A$ a subset of $X$. The $\psi_{i}$-closure and $\psi_{i}$-interior of $A$ with respect to $\psi_{i}$ are denoted by $c_{\psi_{i}}(A)$ and $i_{\psi_{i}}(A)$, respectively, for $i=1,2$.

Theorem 3.9. Let $\left(X, \psi_{1}, \psi_{2}\right)$ be a biGTS and $A \subseteq X$. Then

(1) $c_{q_{\psi}}(A)=c_{\psi_{1}}(A) \cap c_{\psi_{2}}(A)$.

(2) $i_{q_{\psi}}(A)=i_{\psi_{1}}(A) \cup i_{\psi_{2}}(A)$.

(3) $A$ is quasi $q_{\psi}$-closed iff $c_{q_{\psi}}(A)=A$.

(4) $A$ is quasi $q_{\psi}$-open iff $i_{q_{\psi}}(A)=A$. $\emptyset$.

(5) $x \in c_{q_{\psi}}(A)$ iff for every quasi $q_{\psi}$-open set $U$ containing $x, A \cap U \neq$

(6) $x \in i_{q_{\psi}}(A)$ iff there exists a quasi $q_{\psi^{-}}$open set $U$ containing $x$ such that $x \in U \subseteq A$. 
(7) $c_{q_{\psi}}(A)=X-i_{q_{\psi}}(X-A) ; i_{q_{\psi}}(A)=X-c_{q_{\psi}}(X-A)$

Proof. (1) From Lemma 3.5, it follows

$$
\begin{aligned}
c_{q_{\psi}}(A) & =\cap\left\{F: A \subseteq F \text { for a quasi } q_{\psi^{-}} \text {-closed set } F\right\} \\
& =\cap\left\{F: A \subseteq F, \quad F=F_{1} \cap F_{2} \text { for a } \psi_{i} \text {-closed set } F_{i}, i=1,2\right\} \\
& =\cap\left\{F_{1} \cap F_{2}: A \subseteq F_{1} \cap F_{2} \text { for a } \psi_{i} \text {-closed set } F_{i}, i=1,2\right\} \\
& =\left(\cap\left\{F_{1}: A \subseteq F_{1} \text { for a } \psi_{1} \text {-closed set } F_{1}\right\}\right) \\
& \cap\left(\cap\left\{F_{2}: A \subseteq F_{2} \text { for a } \psi_{2} \text {-closed set } F_{2}\right\}\right) \\
& =c_{\psi_{1}}(A) \cap c_{\psi_{2}}(A) .
\end{aligned}
$$

Similarly, (2) is obtained.

(3) If $A$ is quasi $q_{\psi}$-closed, then $A=F_{1} \cap F_{2}$ for a $\psi_{1}$-closed set $F_{1}$ and a $\psi_{2}$-closed set $F_{2}$, and so $c_{\psi_{1}}(A) \subseteq c_{\psi_{1}}\left(F_{1}\right)$ and $c_{\psi_{2}}(A) \subseteq c_{\psi_{2}}\left(F_{2}\right)$. And by (1),

$$
c_{q_{\psi}}(A)=c_{\psi_{1}}(A) \cap c_{\psi_{2}}(A) \subseteq c_{\psi_{1}}\left(F_{1}\right) \cap c_{\psi_{2}}\left(F_{2}\right) \subseteq F_{1} \cap F_{2}=A .
$$

Consequently, $c_{q_{\psi}}(A)=A$.

(4) The proof is similar to that of (3).

(5),(6) It can be easily proved.

(7) From Theorem 2.1, (1) and (2), it follows

$c_{q_{\psi}}(A)=c_{\psi_{1}}(A) \cap c_{\psi_{2}}(A)=\left(X-i_{\psi_{1}}(X-A)\right) \cap\left(X-i_{\psi_{2}}(X-A)\right)=$ $X-\left(i_{\psi_{1}}(X-A) \cup i_{\psi_{2}}(X-A)\right)=X-i_{q_{\psi}}(X-A)$.

Similarly, we can show that $i_{q_{\psi}}(A)=X-c_{q_{\psi}}(X-A)$.

Definition 3.10. Let $\left(X, \psi_{1}, \psi_{2}\right)$ be a biGTS. $X$ is said to be quasi $q_{\psi} T_{1}$ if for $x, y \in X, x \neq y$, there exist quasi $q_{\psi}$-open sets $U$ and $V$ such that $x \in U, y \notin U$ and $x \notin V, y \in V$.

Theorem 3.11. Let $\left(X, \psi_{1}, \psi_{2}\right)$ be a biGTS. Then $X$ is quasi $q_{\psi} T_{1}$ iff singletons are quasi $q_{\psi}$-closed.

Proof. Let $X$ be quasi $q_{\psi} T_{1}$ and $x \in X$. For $y \in X$ with $x \neq y$, there exists a quasi $q_{\psi}$-open set $U_{y}$ such that $y \in U_{y}$ and $x \notin U_{y}$. Then $X-U_{y}$ is a quasi $q_{\psi}$-closed set containing $x$ and so $\cap\left(X-U_{y}\right)=\{x\}$. Hence the singleton set $\{x\}$ is quasi $q_{\psi}$-closed.

The converse is obvious. 
Let $\left(X, \psi_{1}, \psi_{2}\right)$ be a biGTS and $A \subseteq X$. We define the quasi $q_{\psi}$-kernel (briefly $q_{\psi} \operatorname{ker}(A)$ ) as the following:

$$
q_{\psi} \operatorname{ker}(A)=\cap\left\{F: A \subseteq F \text { for a quasi } q_{\psi} \text {-open set } F\right\} .
$$

Theorem 3.12. Let $\left(X, \psi_{1}, \psi_{2}\right)$ be a biGTS. Then the following are equivalent:

(1) $X$ is quasi $q_{\psi} T_{1}$.

(2) For every $A \subseteq X, A=q_{\psi} \operatorname{ker}(A)$.

(3) For $x \in X,\{x\}=q_{\psi} \operatorname{ker}(\{x\})$.

Proof. (1) $\Rightarrow(2)$ Let $A \subseteq X$. For each $x \notin A$, from Theorem 3.11, $X-\{x\}$ is quasi $q_{\psi}$-open and $A \subseteq X-\{x\}$. This implies

$$
A \subseteq q_{\psi} \operatorname{ker}(A) \subseteq \cap_{x \notin A}(X-\{x\})=A .
$$

So $A=q_{\psi} \operatorname{ker}(A)$.

(2) $\Rightarrow(3)$ Obvious.

$(3) \Rightarrow(1)$ For $x, y \in X$ with $x \neq y$, by (3), $\{x\}=q_{\psi} k e r(\{x\})$ and $\{y\}=q_{\psi} \operatorname{ker}(\{y\})$. Since $y \notin q_{\psi} \operatorname{ker}(\{x\})$ and $x \notin q_{\psi} k \operatorname{ker}(\{y\})$, there exist quasi $q_{\psi}$-open sets $U$ and $V$ such that $x \in U, y \notin U$ and $y \in V$, $x \notin V$. Hence $X$ is quasi $q_{\psi} T_{1}$.

\section{4. quasi $\left(q_{\psi}, q_{\mu}\right)$-continuity}

Definition 4.1. Let $\left(X, \psi_{1}, \psi_{2}\right)$ and $\left(Y, \mu_{1}, \mu_{2}\right)$ be two biGTS's. Then a function $f:\left(X, \psi_{1}, \psi_{2}\right) \rightarrow\left(Y, \mu_{1}, \mu_{2}\right)$ is said to be quasi $\left(q_{\psi}, q_{\mu}\right)$ continuous (or quasi generalized continuous) if for every quasi $q_{\mu}$-open set $U$ in $\left(Y, \mu_{1}, \mu_{2}\right), f^{-1}(U)$ is quasi $q_{\psi^{-o p e n ~}}$ in $\left(X, \psi_{1}, \psi_{2}\right)$.

Let $\left(X, \psi_{1}, \psi_{2}\right)$ be a biGTS. The bigeneralized topological space $X$ is said to be strong if $X$ is quasi $q_{\psi^{-o p e n}}$.

Theorem 4.2. Let $f:\left(X, \psi_{1}, \psi_{2}\right) \rightarrow\left(Y, \mu_{1}, \mu_{2}\right)$ be a quasi $\left(q_{\psi}, q_{\mu}\right)$ continuous function on two biGTS's $\left(X, \psi_{1}, \psi_{2}\right),\left(Y, \mu_{1}, \mu_{2}\right)$. If $\left(Y, \mu_{1}, \mu_{2}\right)$ is strong, then $\left(X, \psi_{1}, \psi_{2}\right)$ is also strong.

Proof. Since $Y$ is quasi $q_{\mu}$-open, from quasi $\left(q_{\psi}, q_{\mu}\right)$-continuity of $f$, $f^{-1}(Y)=X$ must be quasi $q_{\psi}$-open. Thus $X$ is strong.

Theorem 4.3. Let $\left(X, \psi_{1}, \psi_{2}\right)$ and $\left(Y, \mu_{1}, \mu_{2}\right)$ be two biGTS's. Then the following are equivalent:

(1) $f$ is quasi $\left(q_{\psi}, q_{\mu}\right)$-continuous.

(2) $f^{-1}\left(i_{q_{\mu}}(B)\right) \subseteq i_{q_{\psi}}\left(f^{-1}(B)\right)$ for all $B \subseteq Y$. 
(3) $c_{q_{\psi}}\left(f^{-1}(B)\right) \subseteq f^{-1}\left(c_{q_{\mu}}(B)\right)$ for all $B \subseteq Y$.

(4) $f\left(c_{q_{\psi}}(A)\right) \subseteq c_{q_{\mu}}(f(A))$ for all $A \subseteq X$.

(5) For every quasi $q_{\mu}$-closed set $F$ in $Y, f^{-1}(F)$ is quasi $q_{\psi}$-closed in $X$.

(6) For each $x \in X$ and each quasi $q_{\mu}$-open set $V$ containing $f(x)$, there exists a quasi $q_{\psi}$-open set $U$ containing $x$ such that $f(U) \subseteq V$.

Proof. (1) $\Rightarrow(2)$ For every $B \subseteq Y$, since $i_{q_{\mu}}(B)$ is quasi $q_{\mu}$-open, by (1), easily we have the condition (2).

(2) $\Rightarrow(1)$ Obvious.

(2) $\Leftrightarrow$ (3) It follows from Theorem 3.9.

$(3) \Leftrightarrow(4)$ and $(1) \Leftrightarrow(5)$ Obvious.

$(6) \Rightarrow(1)$ Let $V$ be a quasi $q_{\mu}$-open set in $\left(Y, \mu_{1}, \mu_{2}\right)$ and $x \in f^{-1}(V)$. Then by (6), there exists a quasi $q_{\psi}$-open set $U_{x}$ containing $x$ such that $f\left(U_{x}\right) \subseteq V$. So by Lemma $3.5, f^{-1}(V)=\cup U_{x}$ is quasi $q_{\psi^{-}}$open. Thus $f$ is quasi $\left(q_{\psi}, q_{\mu}\right)$-continuous.

(1) $\Rightarrow(6)$ Obvious.

\section{References}

[1] Á. Császár; Generalized Topology, Generalized Continuity, Acta Math. Hungar., 96 (2002), 351-357.

[2] M. C. Datta; Contribution to the theory of bitopological spaces, Ph. D. Cissertation, B.I.T. Pilani(India), 1971.

[3] J. C. Kelly; Bitopological spaces, Proc. London Mat. Soc.,13(3)(1963), 71-89.

Department of Mathematics,

Kangwon National University,

Chuncheon, 200-701, Korea

E-mail: wkmin@kangwon.ac.kr

Department of Mathematics,

MyongJi University,

Youngin 449-728, Korea

E-mail: ykkim@mju.ac.kr 